\title{
Combinatorial gene targeting hTERT and BI-1 in CNE-2 nasopharyngeal carcinoma cell line
}

\author{
YAN LIANG ${ }^{*}$, XIANGYONG LI*, RONGWEN LIN* ${ }^{*}$, XIN ZHANG, HUIMIN WANG, \\ NING TAN, KESHEN LI, XUDONG TANG, KEYUAN ZHOU and TAO LI \\ Key Laboratory for Medical Molecular Diagnostics of Guangdong Province, \\ Guangdong Medical College, Dongguan, Guangdong 523808, P.R. China
}

Received August 14, 2012; Accepted October 16, 2012

DOI: $10.3892 /$ br.2012.39

\begin{abstract}
Nasopharyngeal carcinoma (NPC) is a common malignant tumor. In recent studies, we demonstrated that overexpression of the Bax inhibitor-1 (BI-1) induces cell transformation in NIH3T3 cells and that knockdown of BI-1 and human telomerase reverse transcriptase (hTERT) gene expression suppresses NPC cell proliferation and induces apoptosis. To evaluate the combination anti-tumor effects of siRNAs against hTERT and BI-1 in the CNE-2 NPC cell line, combined and separate short-hairpin (sh)RNA plasmids targeting hTERT and BI-1, respectively, were constructed. hTERT and BI-1 mRNA and protein levels were examined by real-time polymerase chain reaction (PCR) and western blot analysis. Cell proliferation, colony formation and migration ability were measured by 3-(4,5-dimethylthiazol2-yl)-2,5-diphenyltetrazolium bromide (MTT), soft agar and wound healing assay. Cell apoptosis was observed by flow cytometry, Hoechst 33258 staining and caspase-3 activity. hTERT, BI-1 and combined shRNA plasmids were injected into xenograft NPC tumor tissues, and expression of hTERT and BI-1 was detected by real-time PCR and immunohistochemistry. Tumor growth was measured by tumor volume and apoptosis in vivo was confirmed by TdT-mediated dUTP nick end-labeling (TUNEL). Our results showed that combined shRNA specific for hTERT and BI-1 markedly suppressed hTERT and BI-1 gene expression in vitro and in vivo. In addition, CNE-2 cell proliferation was inhibited in vitro as well as in vivo. Following the knockdown of the two gene expressions, CNE-2 exhibited a decrease in colony formation and migration ability and an increase in the apop-
\end{abstract}

Correspondence to: Professor Tao Li, Key Laboratory for Medical Molecular Diagnostics of Guangdong Province, Guangdong Medical College, 1 Xincheng Broadway, Dongguan, Guangdong 523808, P.R. China

E-mail: taoliby@hotmail.com

${ }^{*}$ Contributed equally

Key words: human telomerase reverse transcriptase, Bax inhibitor-1, short-hairpin RNA, gene therapy, nasopharyngeal carcinoma totic rate compared to the control groups. Our in vitro and in vivo study showed that the combinative silencing of the two genes enhanced the therapeutic effect compared to the silencing of each individual shRNA. These data suggested that combinatorial gene therapy targeting hTERT and BI-1 may be beneficial as a tumor therapy strategy against human NPCs.

\section{Introduction}

Nasopharyngeal carcinoma (NPC) is a common malignant tumor in southern China, particularly in the Guangdong population (1). Genetic as well as environmental factors are known to contribute to NPC tumorigenesis. At present, the standard treatment (the primary treatments) for NPC is radiotherapy, which in certain cases is combined with chemotherapy. However, the disease relapse and metastatic rate remain high (2). Thus, novel modalities of treatment for NPC are needed. Besides conventional therapies, target strategies aimed to reduce the expression of tumor-related genes have been investigated. Of those, small interfering RNAs (siRNAs) are potentially a curative selection.

RNA interference (RNAi) is a natural process through which the expression of a purpose gene can be knocked down theoretically with high specificity and selectivity RNAi (3). It has been used in numerous malignant cancer gene therapies. In colorectal and gastric cancers, knockdown cell division cycle associated 1 (CDCA1) and CDCA1-kinetochore associated 2 (KNTC2) suppressed cell proliferation and induced apoptosis (4). Downregulation of Wnt 2 and $\beta$-catenin by siRNA suppressed malignant glioma cell growth (5). In addition, chronic lymphocytic leukemia cells induced apoptosis following the silencing of ROR1 and FMOD with siRNA (6).

Telomerase is a specific ribonucleoprotein complex that is often highly expressed in various tumors. The induction of human telomerase reverse transcriptase (hTERT) expression results in activity and is involved in the process of human carcinogenesis (7). Knockdown of hTERT by siRNA inhibits human bladder cancer cells (8), hepatocellular carcinoma cells (9) and oral cancer cell proliferation (10). In NPC, 91\% of cells demonstrate hTERT expression and $85 \%$ exhibit telomerase overexpression (2). Short hairpin (sh)RNA targeted against hTERT inhibits cell viability by regulating 
telomerase activity and its related protein expression in NPC cells (11).

A novel anti-apoptotic gene termed Bax inhibitor-1 (BI-1) has been found to represent a new type of regulator of cell death pathways (12). BI-1 has been found to be overexpressed in several tumors and is involved in tumor progression and malignancy due to its anti-apoptotic properties $(13,14)$. Recently, we demonstrated that overexpression of BI-1 induces cell transformation in NIH3T3 cells, and knockdown of BI-1 and hTERT gene expression by siRNA suppresses NPC cell proliferation and induces apoptosis in vivo and in vitro (15-17).

Since cancer cells are characterized by multiple genetic alterations the single inhibition of one tumor-associated gene might not be sufficient as a therapeutic strategy. In the present study, the combination of the anti-tumor effects of siRNAs against hTERT and BI-1 were evaluated in CNE-2 NPC cell lines. Furthermore, inhibition of tumor growth was observed in subcutaneous NPC xenograft mice.

\section{Materials and methods}

Reagents. Dulbecco's modified Eagle's medium (DMEM) completed medium, fetal bovine serum (FBS), Opti-MEM I medium, TRIzol reagent and Lipofectamine ${ }^{\mathrm{TM}} 2000$ (Lipo) were purchased from Invitrogen (Guangzhou, China). Primary antibody against hTERT, BI- 1 and $\beta$-actin were obtained from Santa Cruz Biotechnology, Inc. (Santa Cruz, CA, USA). Enhanced chemiluminescence substrate was purchased from Thermo Scientific (Guangzhou, China). The restriction enzyme was purchased from New England Biolabs (Beijing, China).

Design and construction of shRNA plasmids. hTERT and BI-1 RNAi target sequences were used in this study: hTERT: 5'-gacgtcttcctacgcttca-3' (GenBank no.: AF015950 Position: 2474-2492), BI-1: 5'-gatcaagattatatctggcactg-3' (GenBank no.: BC036203, Position: 738-760). The hTERT and BI-1 and scramble hairpin-like double-stranded oligo DNA was synthesized by Sangon Biotech (Shanghai, China). The restriction enzyme site EcoRI and KpnI for hTERT and NotI and XbaI for BI-1 were designed on the ends of the oligo DNA. The oligo DNA was cloned in pcDNA3.1(+) (Invitrogen) vector and the correct insert was confirmed by DNA sequence.

Cell culture and transfection. Poorly differentiated human CNE-2 NPC cell line was established and provided by Professor Yi Zeng (18). This cell line was cultured in DMEM completed medium supplemented with $10 \%$ FBS, penicillin (100 U/l) and streptomycin (100 $\mu \mathrm{g} / \mathrm{l})$ (Invitrogen Guangzhou, China) at $37^{\circ} \mathrm{C}$ in a humid incubator with $5 \% \mathrm{CO}_{2}$. Using the Lipofectamine ${ }^{\mathrm{TM}} 2000$ reagent (Invitrogen), CNE-2 cells were transfected with (plasmids pcDNA3.1(+) empty vector, sh-hTERT, sh-scramble hTERT, sh-BI-1, sh-scramble BI-1, sh-hTERT-BI-1 and sh-scramble-hTERT-BI-1), according to the manufacturer's protocol.

Real-time reverse transcription polymerase chain reaction (RT PCR). Primers used in this study were: hTERT: 5'-agtgtctg gagcaagttgcaaag-3' and 5'-cacgacgtagtccatgttca- caatc-3', BI-1: were 5'-atcattgtaaccaatcctgccagac-3' and 5'-agcctcgctctgttgatg tgaa-3', $\beta$-actin: 5'- tggcacccagcacaatgaa-3' and 5'-ctaagtca tagtccgcctagaagca-3. After $48 \mathrm{~h}$ of transfection, total RNA was prepared with TRIzol reagent. Quantitative real-time PCR was performed using One Step SYBR ${ }^{\circledR}$ PrimeScript ${ }^{\circledR}$ RT-PCR kit II (Takara Biotechnology Co., Ltd., Dalian, China), following the manufacturer's instructions on ABI $7500 \mathrm{HT}$ real-time PCR detection system. The results were normalized for the expression of $\beta$-actin.

Western blot analysis. The transfected cells were harvested in RIPA buffer and the protein concentration was determined by Bradford reagent (Bio-Rad, Guangzhou, China). Total protein $(100 \mu \mathrm{M})$ was separated on SDS-PAGE gel and transferred onto a polyvinylidene fluoride membrane (Millipore China Ltd., Guangzhou, China). The membrane was incubated overnight at $4^{\circ} \mathrm{C}$ with primary antibodies (1:200 for hTERT and BI-1, respectively, and 1:500 for $\beta$-actin). The primary antibody for hTERT (sc-7212), BI-1 (sc-12393) and horseradish perodidase-conjugated secondary antibody were purchased from Santa Cruz Biotechnology, Inc. The primary antibody (BSAP0060) for $\beta$-actin was purchased from Bioworld Technology (Shanghai, China). The protein bands were visualized using the enhanced chemiluminescence substrate kit (Thermo, Guangzhou, China). The percentage reduction in band intensity was calculated based on the untreated samples and normalized to $\beta$-actin.

Cell proliferation and apoptosis analysis. CNE-2 cells $\left(5 \times 10^{3}\right)$ were seeded in 96-well plates and incubated at $12 \mathrm{~h}$ for transfection. At $48 \mathrm{~h}$ after transfection, each well was treated with $10 \mu 1$ 3-(4,5-dimethylthiazol-2-yl)-2,5-diphenyltetrazolium bromide (MTT) solution $[10 \mathrm{mg} / \mathrm{ml}$ in phosphate-buffered saline (PBS)] and incubated sequentially at $37^{\circ} \mathrm{C}$. Four hours after incubation, $100 \mu \mathrm{l}$ of dimethyl sulfoxide (DMSO) were added to dissolve the crystals. The plate was oscillated for $10 \mathrm{~min}$ at room temperature and absorbance was measured at 570 and $630 \mathrm{~nm}$. The apoptotic level was performed as previously described (16). Briefly, for Hoechst 33258 fluorescence staining, CNE-2 cells were seeded in 24-well plates and treated with $5 \mu \mathrm{l} 20 \mu \mathrm{g} / \mathrm{ml}$ Hoechst 33258 for $30 \mathrm{~min}$ in the dark. The morphological change of nuclei in CNE-2 cells was analyzed and counted under a fluorescent microscope (magnification, x200) in five different fields to discriminate normal and apoptotic cells. Cells were photographed and the images were processed using the Adobe Photoshop software, version 7.0 (Adobe, CA, USA).

Flow cytometry followed an Annexin V-FITC Apoptosis Detection Kit (Nanjin KeyGen Biotech Co., Ltd., Nanjin, China). Briefly, after $48 \mathrm{~h}$ of plasmid transfection, cells were collected and washed with PBS three times. The cells were suspended in $500 \mu \mathrm{l}$ binding buffer and stained with $5 \mu \mathrm{l}$ Annexin V-FITC and $5 \mu \mathrm{l}$ propidium iodide for $15 \mathrm{~min}$ at room temperature in the dark. The cells were examined using a BD FACSCanto II flow cytometer and analyzed using BD FACSDiva software 6. Caspase-3 activity was determined using a caspase assay kit according to the manufacturer's instructions (Clontech, Mountain View, CA, USA). The samples were read at $405 \mathrm{~nm}$ in a microplate reader. 
A
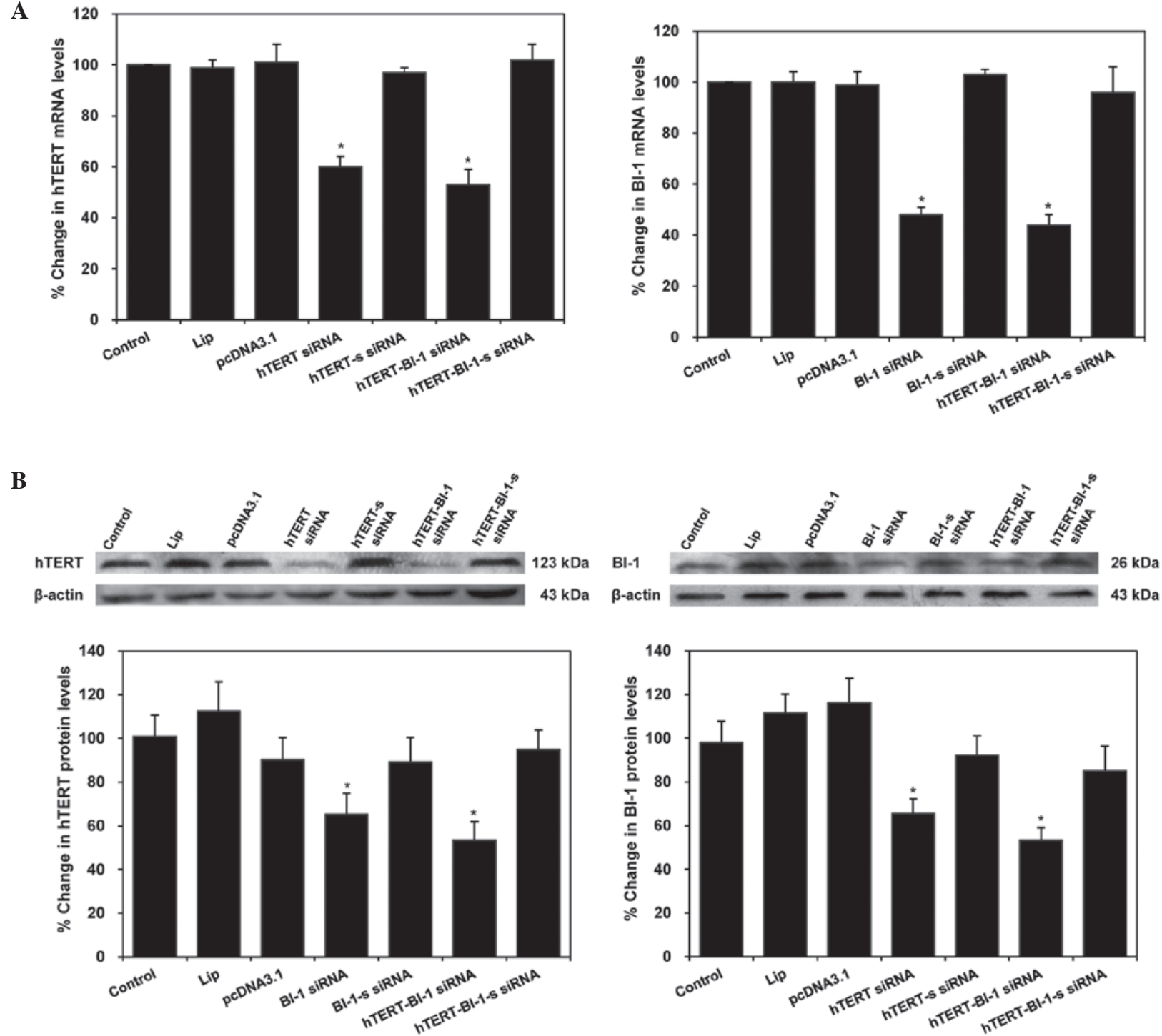

Figure 1. Downregulation of human telomerase reverse transcriptase (hTERT) and Bax inhibitor-1 (BI-1) gene expression after shRNA transfection is shown. hTERT and BI-1 gene expression in CNE-2 cells was suppressed following transfection with sequence-specific short-hairpin (sh)RNA against hTERT, BI-1 and a combination of the two gene plasmids. Untreated, transfection reagent Lipofectamine ${ }^{\mathrm{TM}} 2000$, empty vector pcDNA3.1(+) and vector carrying scrambled shRNA sequence was used as the negative control. After transfection CNE-2 cells were collected and used for RNA and protein isolation, respectively. (A) hTERT and (B) BI-1 mRNA and protein levels were analyzed using real-time PCR and western blot analysis. Results of the analysis are shown in the bar graph. The labels for the bars are: control, untreated, lip transfection reagent Lipofectamine ${ }^{\mathrm{TM}}$ 2000, pcDNA3.1: empty vector pcDNA3.1(+), hTERT or BI-1 siRNA: hTERT- or BI-1-specific shRNA-contained plasmids, hTERT-BI-1 siRNA: hTERT-BI-1 combination shRNA-contained plasmids, hTERT-s, BI-1-s or hTERT-BI-1-s siRNA: hTERT, BI-1 or hTERT-BI-1 scramble shRNA-containing plasmid. The western blot analysis was stripped and re-probed with $\beta$-actin antibody to check for equal loading of total protein. Data are shown as the mean $\pm \mathrm{SD}$ of three independent experiments. "P<0.05 vs. the parental group.

Wound healing assay. Cells $\left(2 \times 10^{5}\right)$ were seeded in a 6-well plate. After forming a confluent monolayer, the cells were transfected with plasmids and scratched using a $100 \mu 1$ tip. The culture medium was replaced with fresh complete medium. The closure of scratch was analyzed under the microscope and images were captured at 0,24 and $48 \mathrm{~h}$ after incubation.

Colony formation assay. Cells $\left(1 \times 10^{4}\right)$ were plated in triplicate in 6 -cm diameter plates with $0.6 \%$ base agar and $0.3 \%$ top agar and incubated for 21 days. The colonies were counted in 10 randomly chosen microscope fields.

In vivo antitumor assay. Twenty BALB/c nude mice (SPF grade, 6-8 weeks of age) were purchased from the Animal
Experimental Center of the Guangdong Medical College. CNE-2 cells $\left(2 \times 10^{6}\right)$ in $100 \mu 1$ serum-free medium were injected into the right flank of each mouse. When the tumors reached $3-5 \mathrm{~mm}$ in diameter ( $\sim 6$ days), the mice were randomized into four groups. The hTERT, BI-1, hTERT-BI-1, scrambled shRNA plasmid DNA $(20 \mu \mathrm{g})$ were respectively injected directly into the tumor, which had been mixed with $40 \mu 1$ Lipo. This treatment was performed three times on days 9, 12 and 15 following CEN-2 cell injection. Tumor size was measured every three days and tumor volume was calculated as $\mathrm{V}=\mathrm{ab}^{2} / 2$, where $\mathrm{a}$ is the length and $\mathrm{b}$ is the width of tumor. At day 18 after cell injection, the animals were sacrificed and the tumors were frozen immediately for future assays. Tissue sections were fixed in $4 \%$ paraformaldehyde and embedded 
in paraffin. Tissue sections $(3 \mu \mathrm{M})$ were prepared and stained with hematoxylin and eosin. The expression levels of hTERT and BI-1 were examined by real-time RT PCR and immunohistochemical staining, performed according to the ImmunoCruz ${ }^{\mathrm{TM}}$ Staining Systems manufacturer's instructions (Santa Cruz Biotechnology, Inc.). Briefly, paraffin sections of tumor tissues were immersed in the primary antibody to hTERT and BI-1 (Santa Cruz Biotechnology, Inc., 1:100) at $4^{\circ} \mathrm{C}$ overnight and detected by a biotinylated secondary antibody (Santa Cruz Biotechnology, Inc.) at room temperature for $1 \mathrm{~h}$. Subsequently, sections were incubated in the HRP-streptavidin complex (Santa Cruz Biotechnology, Inc.) for $30 \mathrm{~min}$. Sections were then incubated with three drops of peroxidase substrate for $12 \mathrm{~min}$ and counterstained with hematoxylin for 5-10 sec, washed, dehydrated in ascending grades of ethanol, and coverslipped with Depex. The sections were examined and microphotographed by light microscope (magnification,x200). Cell apoptosis was determined using the TUNEL method. The animal experiments were approved by the Experimental Animal Care and Use Committee in Guangdong Medical College.

Statistical analysis. Data were shown as the mean \pm standard deviation (SD) and were analyzed using the commercially available statistical software package, SPSS 13.0 (SPSS, IL, USA). One-way analysis of variance (ANOVA) test was performed. $\mathrm{P}<0.05$ was considered to indicate a statistically significant difference.

\section{Results}

Specific knockdown of hTERT and BI-1 in CNE-2 NPC cell line. hTERT- and BI-1-specific inhibited and scrambled shRNA fragments were cloned to mammalian cell expression vector pcDNA3.1(+). The constructs were identified using DNA sequencing and the sequences were correct (data not shown). Plasmid DNA was transfected to CNE-2 cells. After $48 \mathrm{~h}$, the hTERT and BI-1 mRNA and protein expression were detected using real-time PCR and western blot analysis, respectively (Fig. 1). Suppression of mRNA and protein expression was observed (Fig. 1). The combined hTERT/BI-1 shRNA vector simultaneously inhibited hTERT and BI-1 gene expression with the same efficiency as single hTERT or BI-1 shRNA carrying vectors. Untreated, transfection reagent, empty vector and vector-carrying scrambled shRNA sequence was used as the negative controls.

Simultaneous knockdown of hTERT and BI-1 inhibited $C E N-2$ cell growth and migration. Inhibition of the proliferation and migration of CNE-2 cells by hTERT, BI-1 and combined hTERT/BI-1 shRNA was tested through cell viability, soft agar colony formation ability and healing wound assay (Fig. 2). Using the MTT assay, we analyzed in vitro cell growth following hTERT, BI-1 and combined hTERT/BI-1 shRNA-mediated knockdown (Fig. 2A). Significant suppression of cell proliferation was observed in CNE-2 cells at $48 \mathrm{~h}$. The double knockdown group showed better inhibitory effect following reduction of the cell growth rate to $49.00 \pm 3.75 \%$. Results of the colony formation test also showed that cell growth inhibition of CNE-2 cells transfected with hTERT,
BI-1 and combined hTERT/BI-1 shRNA plasmids (Fig. 2B). The colony formation rate was $9.25 \pm 1.05,8.95 \pm 1.17$ and $6.72 \pm 1.00 \%$ for the hTERT, BI-1 and combined hTERT/BI-1 shRNA plasmid transfection groups, respectively. Inhibition of the migration ability for CNE-2 cells was tested following hTERT, BI-1 and combined hTERT/BI-1 shRNA plasmid transfection by healing wound assay. The wound was almost completely repaired after $48 \mathrm{~h}$ in the control group, while the hTERT, BI-1 and combined hTERT/BI-1 shRNA plasmid transfection groups were still in the process of completing the repair (Fig. 2C). Compared to the single knockdown groups, the double-gene knockdown group inevitably showed a decrease in repairing ability.

Induction of apoptosis following shRNA treatments. CNE-2 cells treated with control or hTERT, BI-1 and combined hTERT/ BI-1 shRNA plasmids for $48 \mathrm{~h}$ were evaluated for apoptosis. The apoptosis was determined using Hoechst 33258 fluorescence staining, flow cytometry and caspase- 3 activity. Our results showed that treatment of CNE-2 cells with hTERT, BI-1 and combined hTERT/BI-1 shRNA plasmids for $48 \mathrm{~h}$ resulted in apoptotic bodies and fragmentation of nuclei, as shown by Hoechst 33258 fluorescence staining (Fig. 3A). Flow cytometry showed (Fig. 3B), that the apoptotic rate of the hTERT, BI-1 and combined hTERT/BI-1 shRNA plasmid transfection groups to be $17.20 \pm 2.40,16.73 \pm 2.30$ and $21.90 \pm 1.87 \%$ respectively, which was significantly higher compared to the control group $(3.53 \pm 2.44 \%)$. A more quantitative apoptotic manner was measured by caspase-3 activity, while transfection with hTERT, BI-1 and combined hTERT/BI-1 shRNA plasmids resulted in a $2.7 \pm 0.4-, 2.4 \pm 0.3$ - and 3.7 \pm 0.3 -fold increase in caspase-3 activity (Fig. 3C). Compared with hTERT and BI-1 single blocked groups. The double hTERT/BI-1 blocked group showed an increased apoptotic rate. These findings are consistent with our previous findings and support the hypothesis that double-gene silencing is better than single gene silencing in inducing CNE-2 cell apoptosis.

Proliferation inhibition of NPC BALB/c xenograft tumors with hTERT, BI-1 and combined hTERT/BI-1 shRNA in vivo. After shRNA was injected three times, tumor growth was significantly reduced in hTERT-, BI-1- and combined hTERT/BI-1 shRNA-treated NPC tumors, while no obvious effects were observed on the control group (Fig. 4A). The difference of the average size between the tumors from the hTERT-, BI-1and combined hTERT/BI-1 shRNA-treated groups and the tumors from the control groups was statistically significant on day 18. The average tumor size for the untreated, hTERT-, BI-1- and combined hTERT/BI-1 shRNA-treated groups was $1570.70 \pm 108.50,1119.10 \pm 142.72,1070.32 \pm 160.24$ and $908.52 \pm 234.16 \mathrm{~mm}^{3}$, respectively (Fig. 4B). The inhibition rate for the hTERT shRNA-treated group was $28.75 \pm 2.55 \%$, the BI-1 shRNA-treated group was $31.86 \pm 2.99 \%$ and the combined hTERT/BI-1 shRNA-treated group was $42.16 \pm 5.15 \%$ (Fig. 4C). Morphological similarity of the xenograft to the human NPC was evident on paraffin-embedded sections stained with $\mathrm{HE}$ (Fig. 4D).

hTERT, BI-1 and combined hTERT/BI-1 shRNA inhibited hTERT and BI-1 expression and induced apoptosis in vivo. 
A

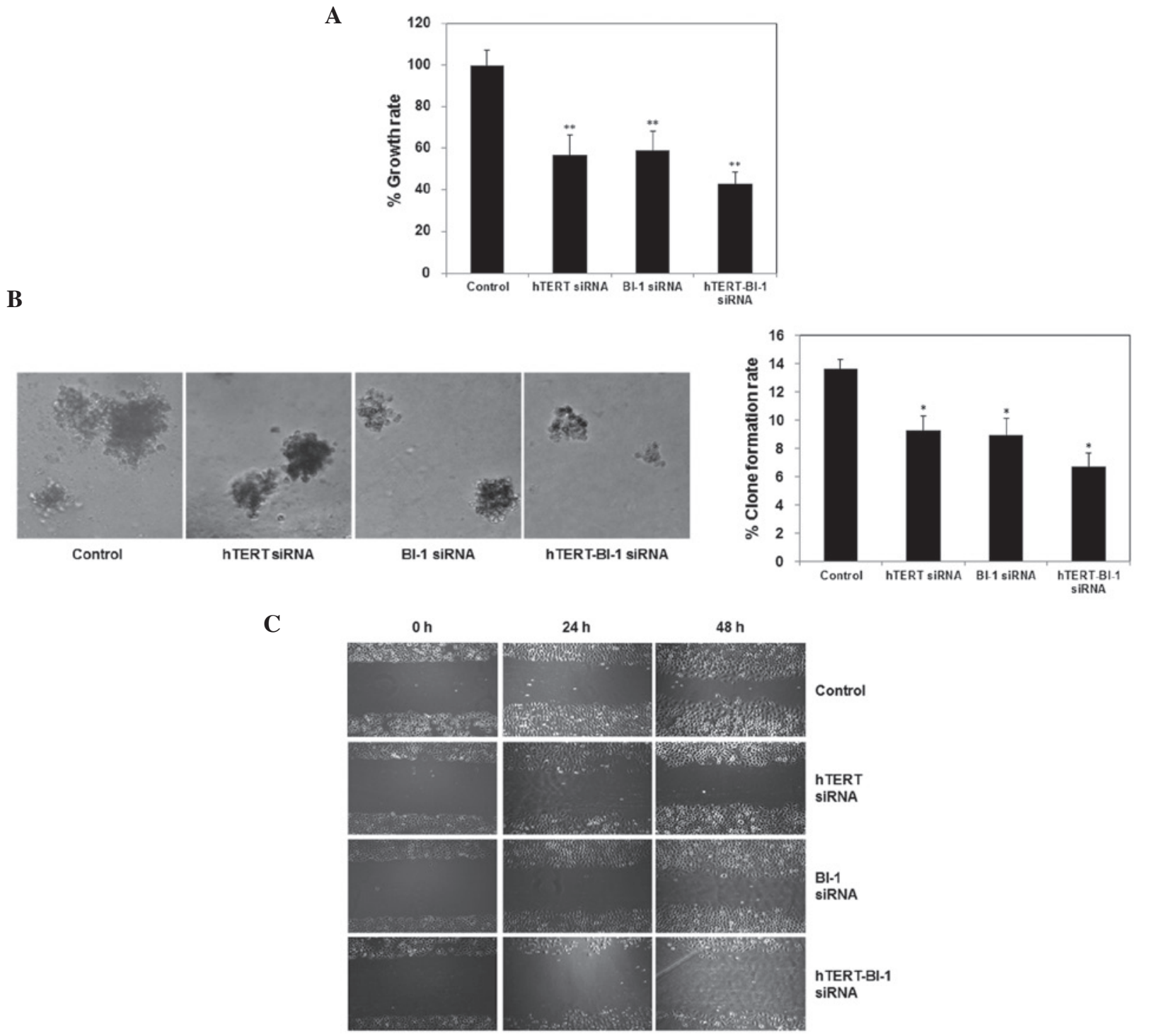

B

Figure 2. Simultaneous knockdown of human telomerase reverse transcriptase (hTERT) and Bax inhibitor-1 (BI-1) inhibited CEN-2 cell growth and migration (A) Cell viability of CNE-2 cells following transfection with shRNA against hTERT, BI-1 or hTERT-BI-1, using MTT assay. The values are normalized to the control (100\%). (B) Colony formation ability of CNE-2 cells following transfection with various plasmids was assayed in soft agar. At day 21, cell colonies were counted in 10 randomly chosen microscope fields and the colony formation rate was calculated. (C) Effect of hTERT, BI-1 or hTERT-BI-1 combinative shRNA on CNE-2 cell migration is shown. CNE-2 cells were seeded in 6-well plates and wounded the following day. Images were captured at 0,24 and $48 \mathrm{~h}$, respectively, after the wound was made. Data were normalized to NS control and are the means \pm SD of triplicates. Asterisks are representative of significant differences compared to the control. $\left({ }^{*} \mathrm{P}<0.05,{ }^{* *} \mathrm{P}<0.01\right)$.

After treatment was administered three times, the mice were sacrificed and the tumor tissues were isolated to detect alterations of hTERT and BI-1 expression. The results (Fig. 5A and B) demonstrated that a significant decrease in the expression of hTERT and BI-1 in tumor tissues treated with hTERT, BI-1 and combined hTERT/BI-1 shRNA compared to the control group. There was no detectable difference in the expression among the lip, empty vector and hTERT, BI-1 hTERT-BI-1 scramble shRNA treatment groups (data not shown).

Cell apoptosis in tumor samples derived from the control and treated mice was examined using the TUNEL method. Results showed that the number of apoptotic cells was markedly increased in the tumor specimens treated with hTERT $(17.34 \pm 2.48 \%)$, BI-1(16.01 $\pm 2.01 \%)$ and combined hTERT/BI-1
$(28.46 \pm 3.28 \%)$ shRNA, as compared to those in tumor specimens from control $(2.87 \pm 1.74 \%)$ or scramble shRNA-treated mice (Fig. 5C).

\section{Discussion}

For NPC, platinum-based doublet chemotherapy is the standard treatment, while targeted approaches are currently being developed (19). Human telomerase is a ribonucleoprotein complex composed of at least the reverse catalytic transcriptase hTERT and the RNA component hTR. The enzyme stabilizes telomere length, thereby contributing to unlimited cell proliferation. Reactivation of telomerase activity during carcinogenesis is a common hallmark in most human tumor types. Previous 
A

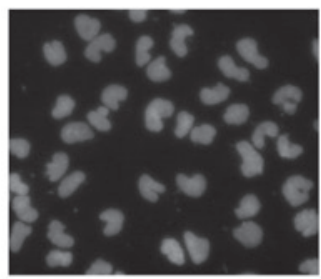

Control

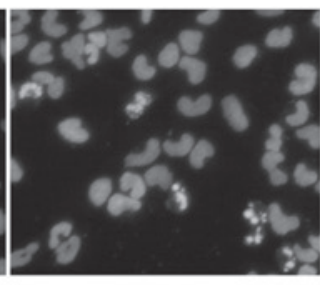

hTERT SiRNA

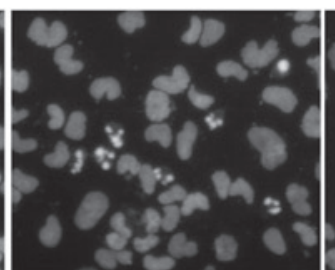

BI-1 siRNA

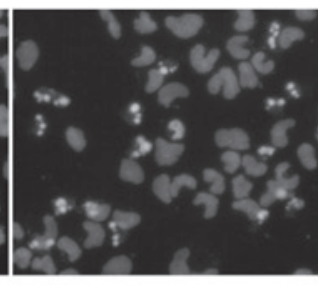

hTERT-BI-1 siRNA

$\mathbf{B}$

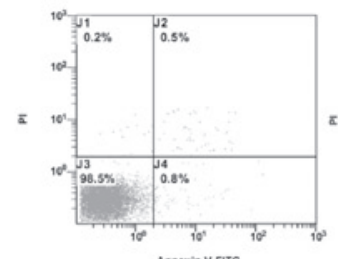

Control

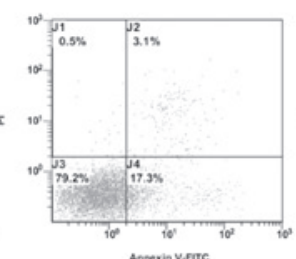

hTERT SIRNA

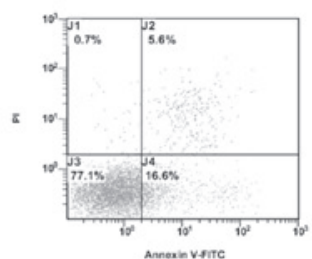

BI-1 siRNA

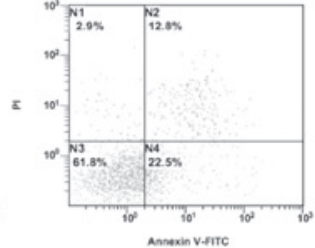

hTERT-BI-1 SIRNA

C
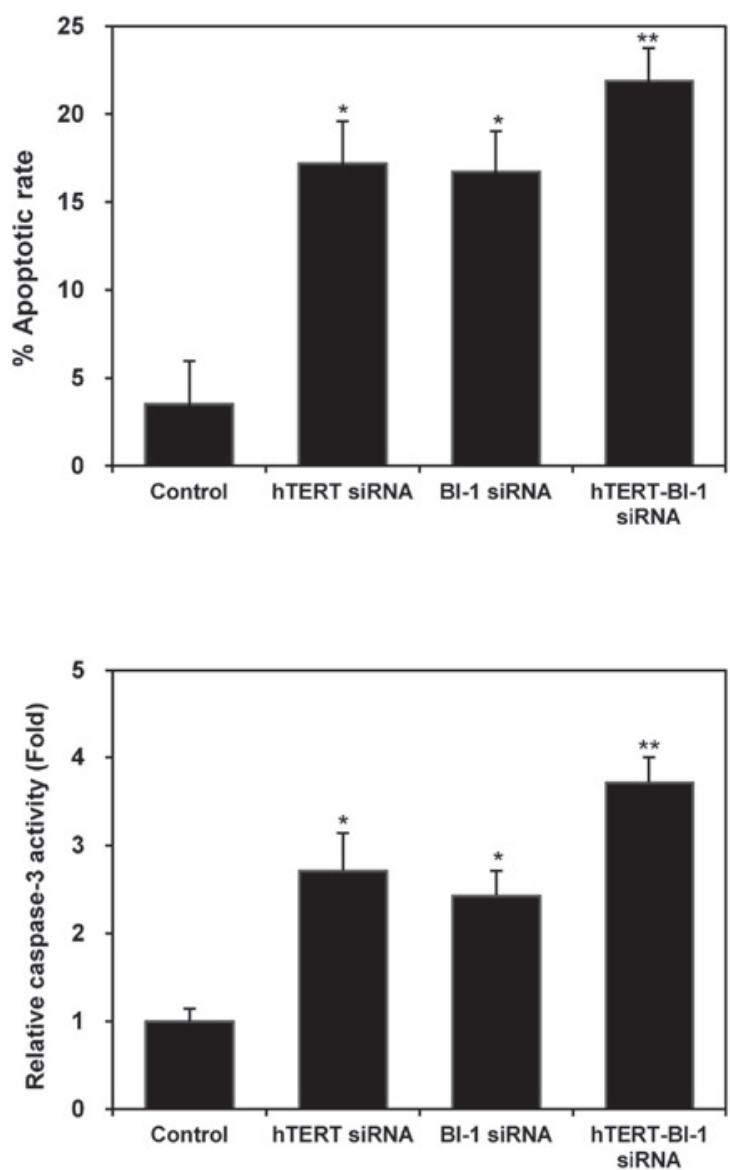

Figure 3. Induction of apoptosis following short hairpin (sh)RNA treatments is shown. (A) Cellular apoptosis was observed using Hoechst 33258 fluorescence staining to detect chromosomal condensation and nuclear fragmentation. (B) Early CNE-2 cells apoptosis rate was analyzed by flow cytometry. (C) Caspase-3 activity was measured and described as a percentage of change in the mean values derived from three separate experiments compared to the control group. Data were normalized to NS control and represent the means \pm SD of triplicates. Asterisks are representative of statistically significant differences compared to the control. $\left({ }^{*} \mathrm{P}<0.05,{ }^{* *} \mathrm{P}<0.01\right)$.

findings have shown that the downregulation of hTERT can lead to a decrease in cell proliferation and/or induction of apoptotic cell death in several types of cancer cells. siRNA inhibited telomerase-induced apoptosis in Barrett's adenocarcinoma cells (20). Similar results were obtained on the human colorectal HT29 cell line (21). In Capan-2 human pancreatic cancer cell knockdown hTERT suppresses cell growth via the inhibition of Bcl-2 and COX-2 expression (22).

The BI-1 family is a highly preserved family of small transmembrane proteins located mostly in the endoplasmic reticulum belonging to the regulatory proteins that determine the fate of a cell regarding death or survival (13). In 
A

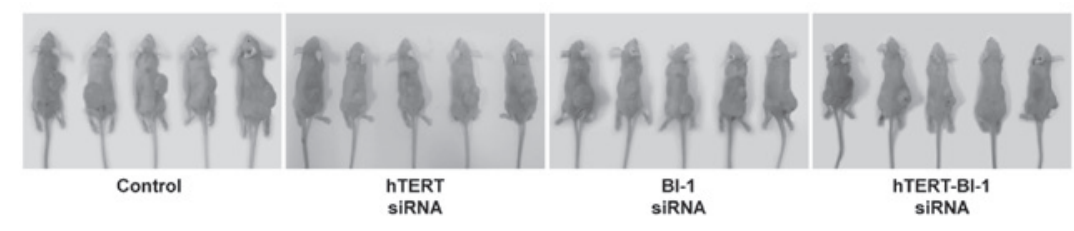

B

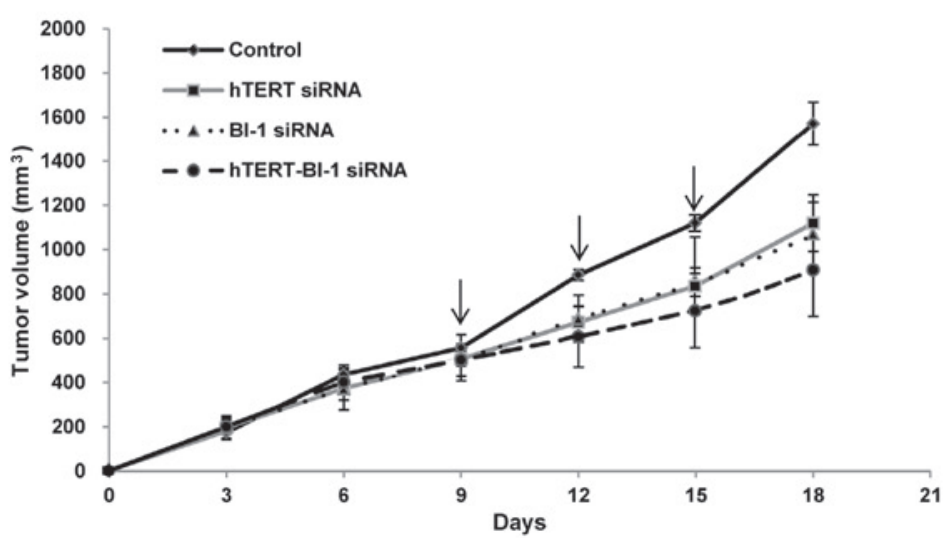

C

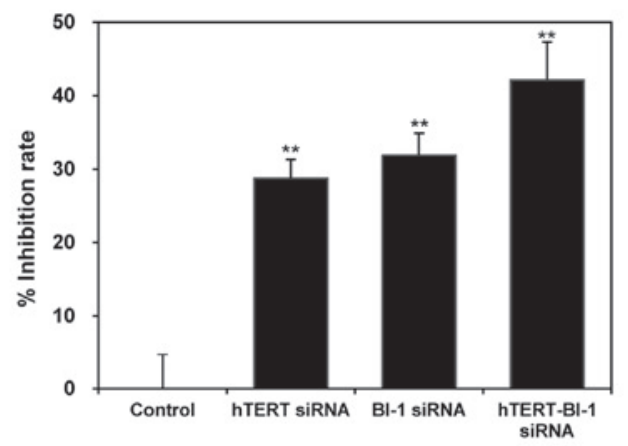

D

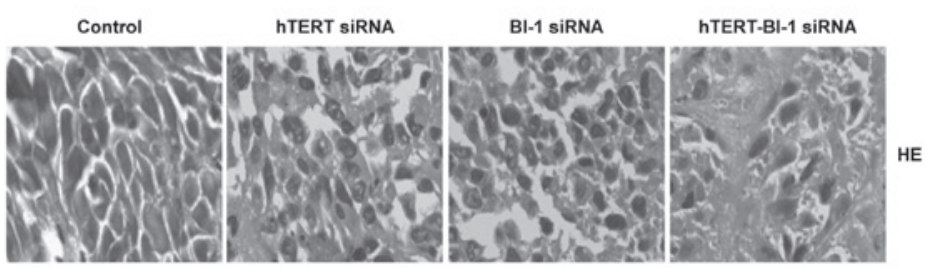

Figure 4. Therapeutic effect of shRNA on the tumor obtained from inoculated CNE-2 cells in nude mice is shown. (A) The appearance of the xenograft subcutaneous nasopharyngeal carcinoma (NPC) in the control and various treatment groups is shown. The xenograft tumors treated with specific targeting short-hairpin (sh)RNA were markedly smaller compared to the control group of mice. (B) Growth curves of subcutaneous NPC in nude mice with various treatments demonstrates that tumor growth was markedly retarded compared to the control group. Compared to the single targeting group, the combinative shRNA-treated group showed enhanced therapeutic effects. (C) Inhibition rate of the control and various treatment groups is shown. As compared to the control group, the inhibitory rate of the three shRNA treatment groups was markedly increased and the combinative shRNA treatment group showed a higher inhibitory effect $\left({ }^{* *} \mathrm{P}<0.01\right)$. (D) Hematoxylin and eosin (HE) staining results of the tumors formed in nude mice, which are typical nasopharyngeal squamous cell carcinomas.

the lung cancer cell lines, HT1080 and B16F10, BI-1 altered mitochondrial function, which in turn altered glucose metabolism and activated sodium-hydrogen exchanger to enhance cancer metastasis. Similar to previous studies, results of our previous studies demonstrated that BI-1 mediated apoptotic resistance in human NPC cells (16). Since tumor cells may bypass the inhibition of a single gene by changing their expression profile, the combined antisense-mediated inhibition of tumor-promoting genes is a promising strategy for cancer treatment.
Multitarget siRNA inhibition of antiapoptotic genes in bladder cancer cells reduced cell growth and sensitized cells to subsequent chemotherapy (23). Recently, Song et al (24) constructed a shRNA plasmid containing VEGF-, C-myc-, survivin- and hTERT-inhibited siRNA sequences and found that the multiple gene shRNA more markedly induced cell apoptosis compared to each individual shRNA, respectively. The combinative silencing of these four genes had an enhanced inhibitory effect on xenograft tumors compared to the silencing of each individual shRNA. The RNA polymerase III promoters 
A

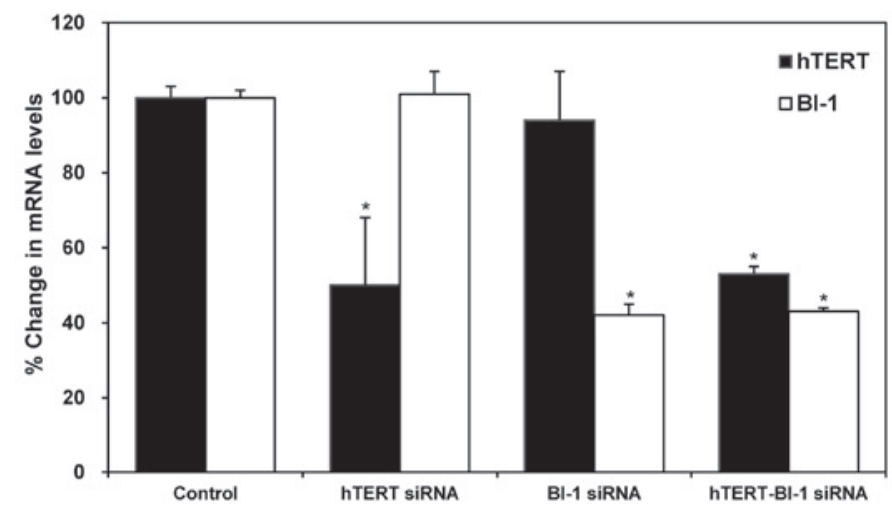

B

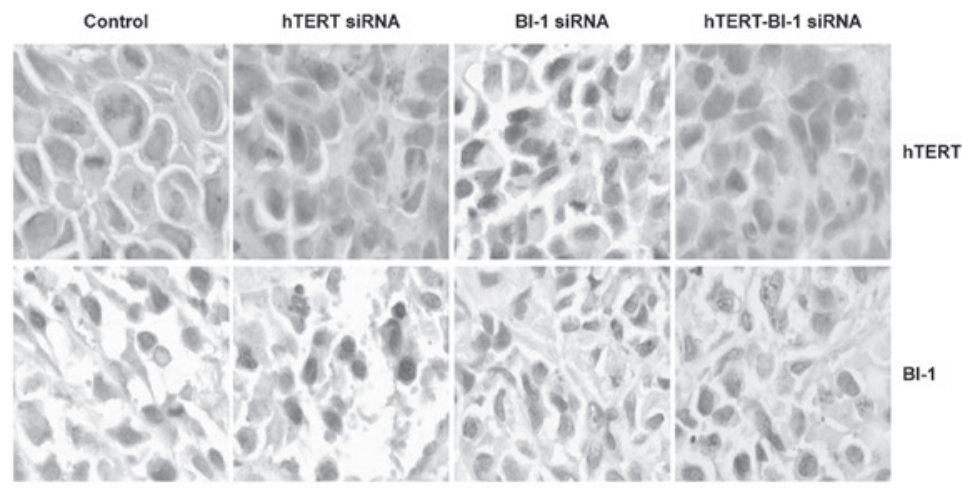

C
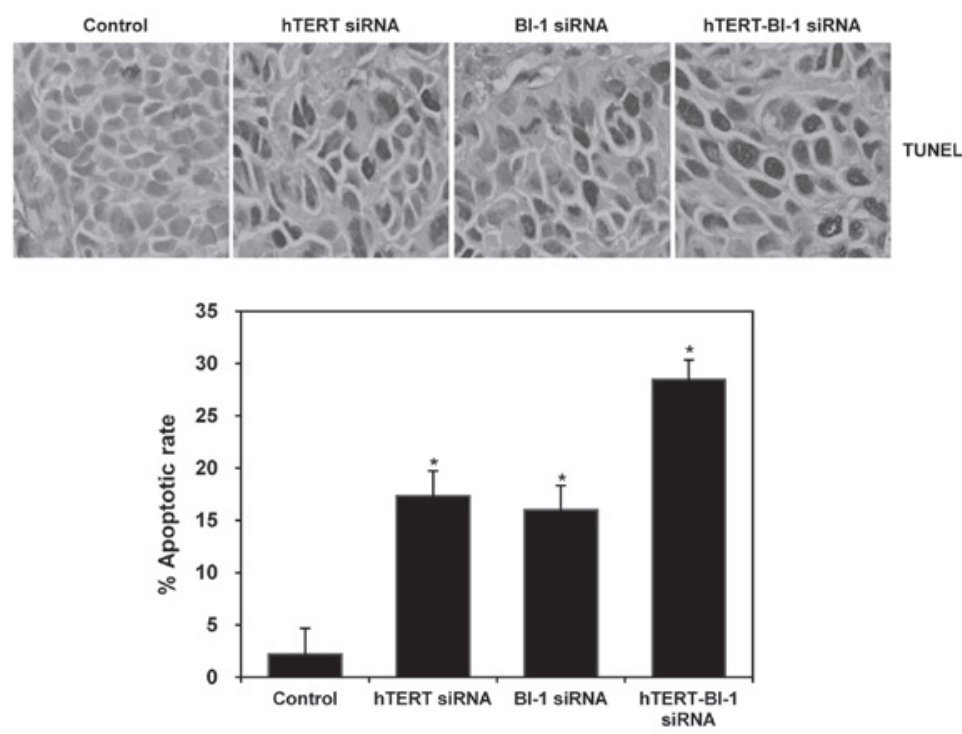

Figure 5. Specific short-hairpin (sh)RNA downregulated hTERT and BI-1 gene expression and induced apoptosis in tumor tissue are shown. (A) Expression of hTERT and BI-1 in subcutaneous nasopharyngeal carcinomas (NPCs) treated with shRNA targeting hTERT or BI-1 was suppressed, as shown by results of real-time RT-PCR. (B) Similar results were also obtained by immunohistochemistry (magnification, $\mathrm{x} 200)($ ( $\mathrm{P}<0.05)$. Compared to the single knockdown group, combinative shRNA simultaneously suppressed hTERT and BI-1 gene expression. (C) Apoptotic cells were increased in subcutaneous NPCs that were treated with shRNA targeting hTERT, BI-1 or combined hTERT/BI-1 detected by the TUNEL (TdT-mediated dUTP nick end-labeling) method (magnification, $\mathrm{x} 200)$ Combinative shRNA-treated group demonstrated a higher apoptotic rate ( $\mathrm{P}<0.05)$.

contained plasmids that synthesized shRNAs and elicit RNAi efficiently, while lacking cell specificity and rendering the monitoring of shRNA expression levels difficult. An alternative method to deliver RNAi is to use RNA polymerase II through direct synthesis of shRNA (25). In this study, we demonstrated that shRNA ligation into vector pcDNA3.1(+) against hTERT and BI-1 led to a notable reduction of gene expression in vitro and in vivo (Fig. 1). The specific suppression of hTERT and BI-1 expression induced a significant decrease in CNE-2 cell proliferation, colony formation and migration (Fig. 2). It also enhanced CNE-2 apoptosis as assessed by flow cytometry, caspase-3 activity and Hoechst 33258 fluorescence staining (Fig. 3). Furthermore, we observed an effective suppression of hTERT and BI-1 expression in the nude mice model. After 
the knockdown of hTERT and BI-1 gene expression simultaneously or respectively by direct neoplasm injection, the xenograft tumor showed retarded growth. Compared to the single knockdown group, the combined knockdown demonstrated a higher inhibition rate (Fig. 4). Moreover, the same effect of apoptosis was confirmed on the xenograft tumor using the TUNEL method (Fig. 5).

In conclusion, to the best of our knowledge, we have demonstrated for the first time that the simultaneous knockdown of hTERT and BI-1 reduced NPC cell proliferation and migration and induced apoptosis in vitro and in vivo. The combination inhibition may greatly improve the outcome of NPC treatment and contribute towards a better clinical prognosis. Therefore, further studies are required to apply these targeted therapies in patients to improve the outcome of NPC treatment.

\section{Acknowledgements}

The authors would like to thank Professor Yi Zeng from the Institute of Virology, China Institute of Preventive Medical Science, for kindly providing the human NPC CNE-2 cell line and Professor Jindong Ni from the Guangdong Medical College for data analysis. This study was supported by the National Natural Science Foundation of China (81172067), the Natural Science Foundation of Guangdong, China (8252402301000001), the Dongguan Higher Education Science and Technology Program (200810815294, 200910815200318 and 201110815200134) and the Dongguan Medical Institutes Science and Technology Program (201110515200499).

\section{References}

1. Parkin DM and Muir CS: Cancer incidence in five continents Comparability and quality of data. IARC Sci Publ: 45-173, 1992

2. Chou J, Lin YC, Kim J, et al: Nasopharyngeal carcinoma - review of the molecular mechanisms of tumorigenesis. Head Neck 30: 946-963, 2008

3. Ichim TE, Li M, Qian H, et al: RNA interference: a potent tool for gene-specific therapeutics. Am J Transplant 4: 1227-1236, 2004.

4. Kaneko N, Miura K, Gu Z, et al: siRNA-mediated knockdown against CDCA1 and KNTC2, both frequently overexpressed in colorectal and gastric cancers, suppresses cell proliferation and induces apoptosis. Biochem Biophys Res Commun 390: $1235-1240,2009$.

5. Pu P, Zhang Z, Kang C, et al: Downregulation of Wnt2 and beta-catenin by siRNA suppresses malignant glioma cell growth. Cancer Gene Ther 16: 351-361, 2009.

6. Choudhury A, Derkow K, Daneshmanesh AH, et al: Silencing of ROR1 and FMOD with siRNA results in apoptosis of CLL cells. Br J Haematol 151: 327-335, 2010.

7. Li Y, Li M, Yao G, et al: Telomerase inhibition strategies by siRNAs against either hTR or hTERT in oral squamous cell carcinoma. Cancer Gene Ther 18: 318-325, 2011.
8. Kraemer K, Fuessel S, Schmidt U, et al: Antisense-mediated hTERT inhibition specifically reduces the growth of human bladder cancer cells. Clin Cancer Res 9: 3794-3800, 2003.

9. Zhang PH, Zou L and Tu ZG: RNAi-hTERT inhibition hepatocellular carcinoma cell proliferation via decreasing telomerase activity. J Surg Res 131: 143-149, 2006.

10. Liu X, Huang H, Wang J, et al: Dendrimers-delivered short hairpin RNA targeting hTERT inhibits oral cancer cell growth in vitro and in vivo. Biochem Pharmacol 82: 17-23, 2011.

11. Wang Y, Duan HG, Chen SM, Xiao BK, Cheng J and Tao ZZ: Effect of RNA interference targeting human telomerase reverse transcriptase on telomerase and its related protein expression in nasopharyngeal carcinoma cells. J Laryngol Otol 121: 476-482, 2007.

12. Xu C, Bailly-Maitre B and Reed JC: Endoplasmic reticulum stress: cell life and death decisions. J Clin Invest 115: 2656-2664, 2005.

13. Reimers K, Choi CY, Bucan V and Vogt PM: The Bax Inhibitor-1 (BI-1) family in apoptosis and tumorigenesis. Curr Mol Med 8: 148-156, 2008.

14. Robinson KS, Clements A, Williams AC, Berger CN and Frankel G: Bax Inhibitor 1 in apoptosis and disease. Oncogene 30: 2391-2400, 2011.

15. Xiang-yong L, Yang-chao C, Ke-yuan Z, Mei-hong Z, Hai-qing L, Hsiang-fu K and Xin Z: Overexpression of Bax inhibitor-1 (BI-1) induces cell transformation in NIH3T3 cells. Cell Biol Int 34: 1099-1104, 2010.

16. Zhang M, Li X, Zhang Y and Zhou K: Bax inhibitor-1 mediates apoptosis-resistance in human nasopharyngeal carcinoma cells. Mol Cell Biochem 333: 1-7, 2010.

17. Li XY, Lai YK, Zhang JF, et al: Lentivirus-mediated RNA interference targeting Bax inhibitor-1 suppresses ex vivo cell proliferation and in vivo tumor growth of nasopharyngeal carcinoma. Hum Gene Ther 22: 1201-1208, 2011

18. Sizhong Z, Xiukung G and Yi Z: Cytogenetic studies on an epithelial cell line derived from poorly differentiated nasopharyngeal carcinoma. Int J Cancer 31: 587-590, 1983.

19. Chan AT: Nasopharyngeal carcinoma. Ann Oncol 21 (Suppl 7): 308-312, 2010.

20. Shammas MA, Koley H, Batchu RB, et al: Telomerase inhibition by siRNA causes senescence and apoptosis in Barrett's adenocarcinoma cells: mechanism and therapeutic potential. Mol Cancer 4: 24,2005

21. de Souza Nascimento P, Alves G and Fiedler W: Telomerase inhibition by an siRNA directed against hTERT leads to telomere attrition in HT29 cells. Oncol Rep 16: 423-428, 2006.

22. Zhong YQ, Xia ZS, Fu YR and Zhu ZH: Knockdown of hTERT by SiRNA suppresses growth of Capan-2 human pancreatic cancer cell via the inhibition of expressions of Bcl-2 and COX-2. J Dig Dis 11: 176-184, 2010.

23. Kunze D, Wuttig D, Fuessel S, et al: Multitarget siRNA inhibition of antiapoptotic genes (XIAP, BCL2, BCL-X(L)) in bladder cancer cells. Anticancer Res 28: 2259-2263, 2008.

24. Song Y, Dong MM and Yang HF: Effects of RNA interference targeting four different genes on the growth and proliferation of nasopharyngeal carcinoma CNE-2Z cells. Cancer Gene Ther 18: 297-304, 2011.

25. Zhou H, Xia XG and Xu Z: An RNA polymerase II construct synthesizes short-hairpin RNA with a quantitative indicator and mediates highly efficient RNAi. Nucleic Acids Res 33: e62, 2005. 